\title{
Control of high-order harmonics for attoscience using a static-electric-field pattern
}

\author{
Carles Serrat \\ UPC-Universitat Politècnica de Catalunya, Colom 11, E-08222 Terrassa (Barcelona), Spain
}

(Received 16 September 2011; published 27 December 2011)

\begin{abstract}
Quantum control in high-order-harmonic generation is considered theoretically by using a spatial distribution of static electric fields along the propagation direction of the driving field. It is shown that the trajectories of the electrons during its acceleration by the laser field in the high-harmonics-generation process can be controlled by periodically distributed static electric fields, which conveniently shape the driving laser field during propagation. Applying this mechanism, a quasi-phase-matching scheme that leads to filtered enhanced high harmonics is achieved. The harmonics in the plateau region are enhanced due to periodical phase variations in the long quantum trajectories as a consequence of the faster change experienced by the intensity-dependent phase along the longer electron trajectories. This effect should be observed in all quasi-phase-matching schemes based on perturbation of the microscopic quantum phase. The richness of adding a static-electric-field spatial pattern in the interaction region suggests a general scheme for feedback loop control in high-order-harmonic generation.
\end{abstract}

DOI: $10.1103 /$ PhysRevA.84.061803

PACS number(s): 42.65.Ky, 37.10.Jk, 32.80.Fb, 32.80.Rm

The possibility of controlling quantum processes occurring in atoms and molecules has been considered for years, especially since the birth of laser physics. Recently highintensity ultrashort phase-stable laser pulses have extended this research field opening the possibility of measuring and controlling attosecond time-scale dynamics of electrons [1]. In this context, the importance of combining schemes and techniques from the quantum control and attoscience scientific communities has emerged naturally. In this Rapid Communication I present a scheme for quantum coherent control in highorder harmonics and attosecond pulse generation which is based on shaping the driving laser field by adding static electric fields at each spot of the interaction region. An electric field whose strength is constant in the time scale of the nonlinear high-harmonics-generation process can be implemented by using a pulsed long-wavelength laser propagating orthogonal to the driving field. This method is all-optical and allows for control of propagation effects.

In order to demonstrate the capability of the control scheme, I consider a configuration consisting of static electric fields periodically distributed along the propagation direction of the driving laser. This simple pattern allows us to observe the effect of the static electric field on the two electron wave-packet trajectories most contributing to the highharmonics-generation (HHG) process, namely, the so-called short and long trajectories. I will show how coherent control of the microscopic interaction between the driving laser and the medium during propagation leads to efficient control of the yield of the generated harmonics by means of a quasi-phase-matching (QPM) macroscopic effect. It is hence not meaningless to say that by tailoring the geometry of the interaction volume, the strength and phase of the driving laser field, together with the amplitudes, length, and distribution of the zones over which the static field is added, optimal control of the microscopic response along the propagation region might be accomplished to obtain the desired properties of the generated harmonics and attosecond pulses, ideally taking

\footnotetext{
*carles.serrat-jurado@upc.edu
}

advantage of learning loop techniques widely used in quantum control [2].

In a previous paper a QPM effect was discussed by considering a static electric field periodically distributed in the interaction volume along the direction of the fundamental driving laser and oriented in the same direction as its polarization [3]. In this paper it was shown that the periodicity of the static-electric-field pattern can be used as a control parameter for tuning the enhancement of the harmonics yield between the extreme ultraviolet and the soft-x-ray regions of the electromagnetic spectrum, predicting an enhancement effect of three orders of magnitude in some cases. In the present Rapid Communication this simple periodic static-electric-field configuration is considered as an example for showing how the added static electric fields control the phase in the short and long quantum electron trajectories generating the harmonics, and how this results in a macroscopic QPM effect. As it will be shown, only the long trajectories contribute to the enhancement of the harmonics on axis in the plateau region, which leads to filtering of the two main path contributions.

In the present simulations a linearly polarized laser pulse with Gaussian temporal and spatial distributions of $5 \mathrm{fs}$ and $40 \mu \mathrm{m}$ diameter of focus [full width at half maximum (FWHM)], central wavelength of $800 \mathrm{~nm}$, and peak intensity at

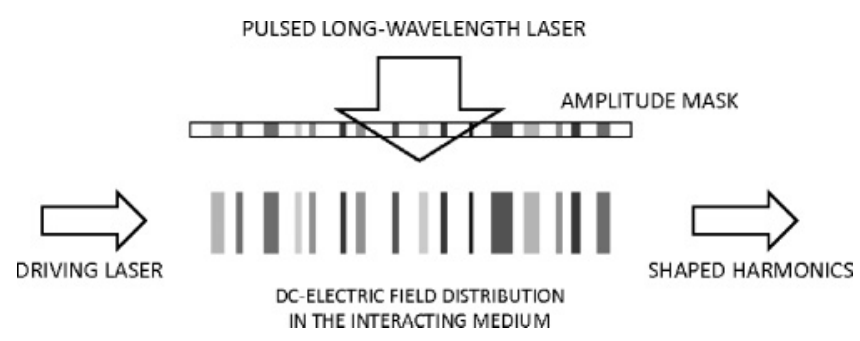

FIG. 1. Schematic illustration of a static electric field distributed along the driving pulse propagation direction. A pulsed longwavelength laser,- such as, e.g., a pulsed terahertz or $\mathrm{CO}_{2}$ laser, is sent through an amplitude mask whose amplitude pattern generates the desired field distribution. Both the long-wavelength laser and the driving laser are linearly polarized in the direction orthogonal to the plane of the figure. 
focus $7 \times 10^{14} \mathrm{~W} / \mathrm{cm}^{2}$ has been considered. This pulse propagates from the focus into a gas medium producing high harmonics with a photon energy cutoff at $\approx 160 \mathrm{eV}$. The medium that has been chosen in the calculations is neon (ionization potential $21.56 \mathrm{eV}$ ) at $20 \mathrm{mbar}$. As schematically illustrated in Fig. 1, the proposed control scheme is based on the presence of a pulsed long-wavelength laser propagating orthogonal to the fundamental beam in the interaction region, both longwavelength and driving lasers being linearly polarized in the same direction. The spatial pattern of the static electric field can be achieved using an amplitude mask on the transverse profile of the long-wavelength laser. This is indeed an appropriate configuration since, on one hand, the carrier electric field of the long-wavelength laser provides a very good approximation of a static electric field in the interaction volume, and, on the other hand, the short duration of the long-wavelength pulse prevents a dielectric breakdown in the medium which would occur due to the relatively high static field strengths considered. The numerical simulations are based on a threedimensional propagation model in cylindrical coordinates using the nonadiabatic strong field approximation [4] to calculate the atomic response, as outlined in Ref. [5], including in the present calculations the absorption and dispersion of the harmonics in the medium. Although the applied static electric field essentially breaks the cylindrical symmetry in the medium, hyperpolarizabilities of an inert gas such as neon at low pressures can be neglected for the propagation distances considered here [6], and thus cylindrical symmetry remains an ideal approximation. The input laser field can be written as $E=E_{0} \exp \left[-(r / \rho)^{2}\right] \exp \left[-(t / \tau)^{2}\right] \cos \left(\omega_{L} t+\phi\right)$, where $E_{0}$ is the peak amplitude at focus, $r$ and $t$ are the radial and temporal variables, $\rho$ and $\tau$ provide the width of the transverse and temporal profiles, respectively, $\omega_{L}$ is the central angular frequency of the laser, and $\phi$ the initial phase, which is taken as null in the present study. The periodic static-electric-field distribution is numerically implemented by adding a constant value $\xi \beta E_{0}$ to the laser field, distributed such that $\xi=0$ at $2 n \Delta_{s}<z<(2 n+1) \Delta_{s}$ and $\xi=1$ elsewhere, with $z$ being the longitudinal coordinate and $n=0,1,2, \ldots$. The strength of the static electric field is thus defined by the parameter $\beta$, which is the fraction of the laser peak amplitude at focus, and $\Delta_{S}$ is half the period of the static-electric-field distribution.

Figure 2 shows the spectrum of the harmonics generated on axis after a propagation distance of $0.5 \mathrm{~mm}$. The dotteddashed black line corresponds to $\beta=0$, and the other lines show the calculations for a static-electric-field strength such as $\approx 3.6 \mathrm{MV} / \mathrm{cm}(\beta=0.005)$ spatially distributed along the propagation direction, with $\Delta_{s}=11 \mu \mathrm{m}$. The dashed gray line corresponds to the enhanced photon yield considering all recombination processes in the numerical simulation, while the solid red (medium gray) line shows the contribution of the long trajectories and the dotted blue (dark gray) line the enhanced yield due to the short trajectories. It is clear that only the contribution of the long trajectories on the harmonics is enhanced in the plateau region $(\approx 80-115 \mathrm{eV})$, while both paths contribute similarly in the cutoff region-which is expected since the duration of the two trajectories approach each other in that region. The contributions of the short and long trajectories for $\beta=0$ are not distinct concerning the harmonics yield (not shown in the figure).

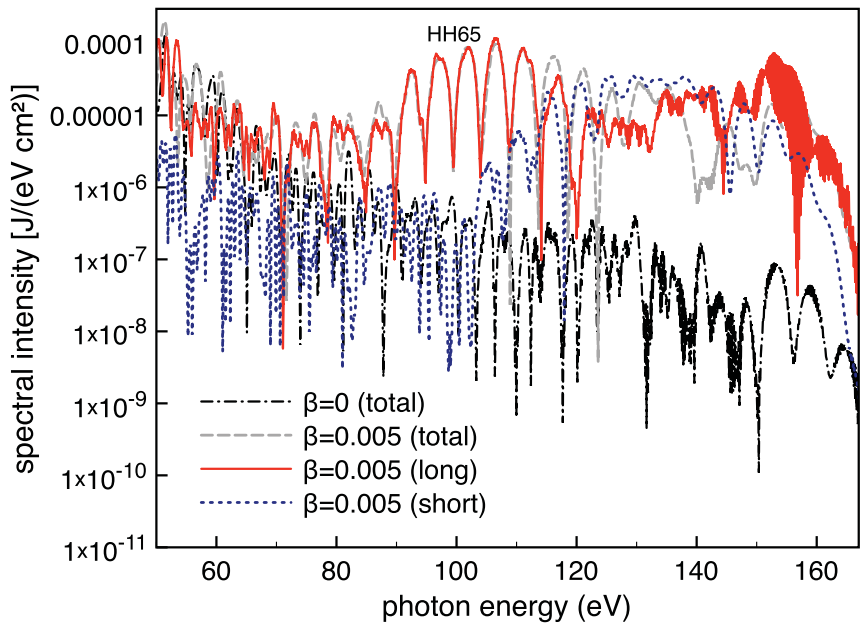

FIG. 2. (Color online) Spectral intensity of the harmonics on axis vs photon energy showing yield enhancement in the plateau and cutoff regions after a propagation distance of $0.5 \mathrm{~mm}$, with $E_{0} \approx$ $726 \mathrm{MV} / \mathrm{cm}$.

In order to further characterize the yield of the harmonics generated by the two distinct electron trajectories, I perform a time-frequency analysis of the harmonics on axis, considering the contribution of all trajectories at a propagation distance of $352 \mu \mathrm{m}$, which is shown in Fig. 3. In the absence of static electric field $(\beta=0)$ a symmetric plot is expected [Fig. 3(a)]. Although the resolution of the figure is not optimal due to limitations from the numerical simulations, the cycles in the photon energy versus time plane, which correspond to the time-dependent intensity profile of the driving laser pulse,

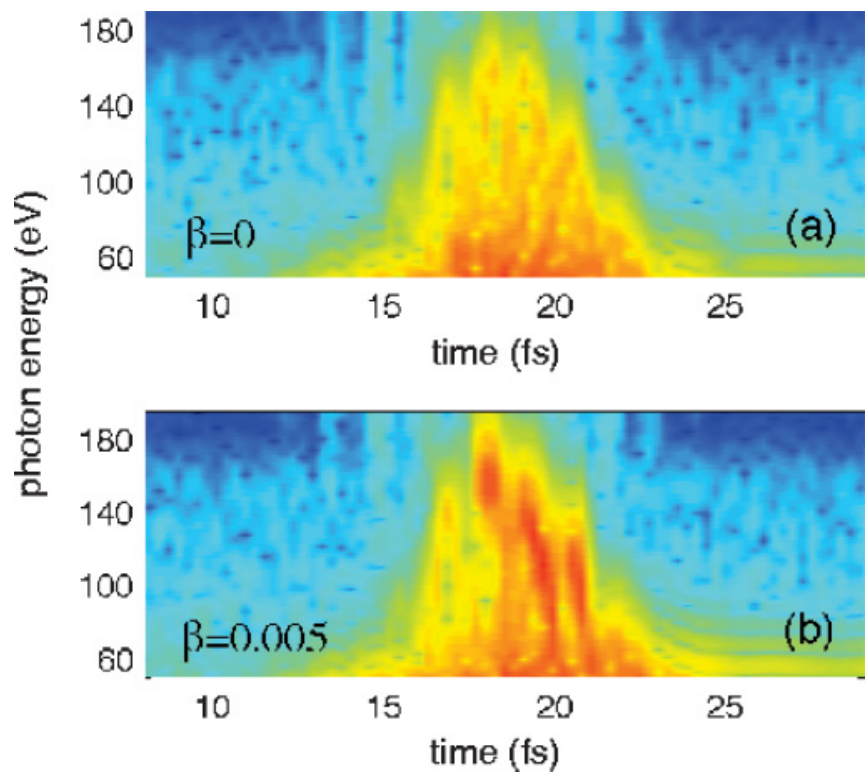

FIG. 3. (Color online) Time-frequency distribution of the harmonics on axis at a propagation distance of $352 \mu \mathrm{m}$. A more intense red (gray) color indicates a higher harmonics yield (only qualitative results are shown for clarity). (a) The case for $\beta=0$ shows no distinction between long- and short-path contributions, while for $\beta=0.005$, in (b), the harmonics of the plateau region are clearly enhanced only due to the contribution of the long paths. 
can still be well recognized. Indeed, each cycle accords to half period of the driving $800-\mathrm{nm}$ pulse, with the earlier half of each cycle showing the first occurring recollisions (short trajectories) and the second half showing the contribution of the long trajectories. The duration of the two trajectories coincides in the cutoff, where the highest photon energies are generated (see Fig. 3). Note that, concerning the yield of the harmonics, only qualitative results are shown in Fig. 3 for clarity, with a more intense red (gray) color indicating a higher harmonics yield. In Fig. 3(b) the static-electric-field distribution is set to $\beta=0.005$. We can clearly observe how the harmonics in the cutoff region are produced by both the short and the long trajectories [relative photon energy maxima in Fig. 3(b)], as expected, while in the plateau region $(\approx 80$ $115 \mathrm{eV}$ ) only the long trajectories contribute to enhance the yield.

Let us now consider in more detail the effect of the static electric field on the microscopic single-atom interaction. A static electric field has been proven previously to be a very sensitive means for controlling the generation of high-order harmonics in the single-atom case. Several important effects have been reported in this context, as, for instance, the enhancement of the yield of the harmonics on the plateau and the extension of the plateau beyond the usual cutoff
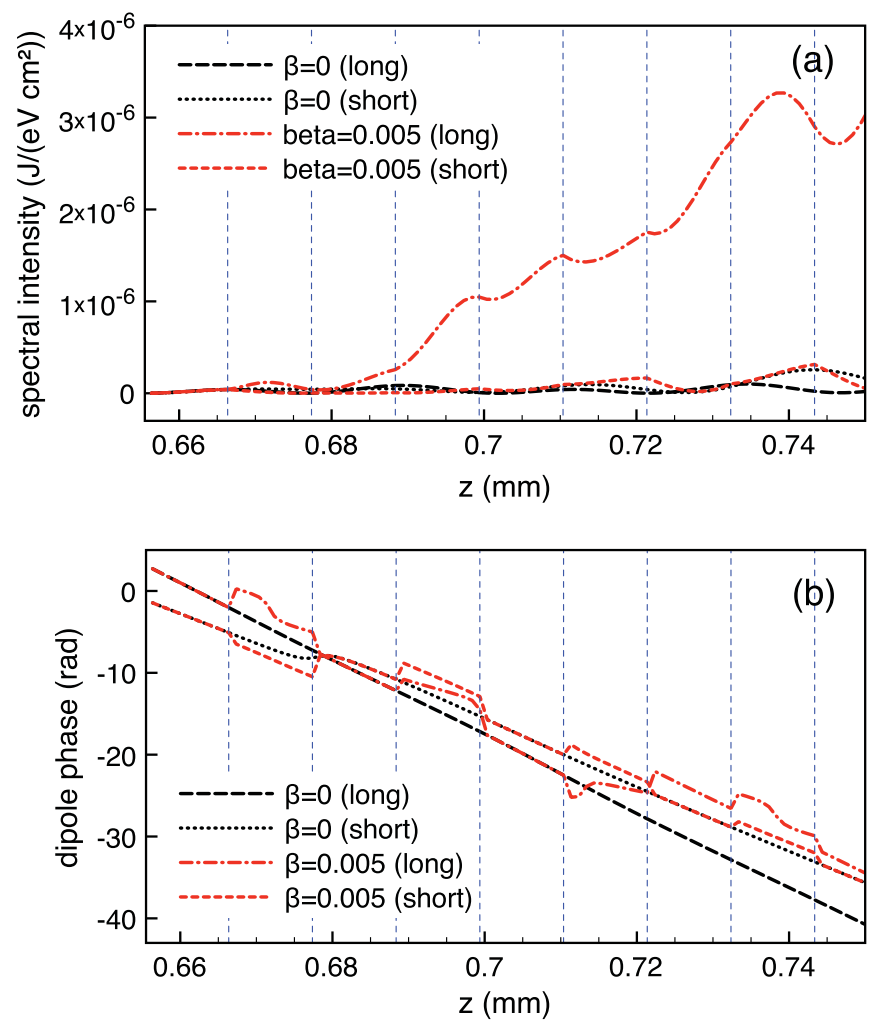

FIG. 4. (Color online) (a) Evolution of the spectral intensity for the long- and short-path contributions, as indicated, in the first $100 \mu \mathrm{m}$ of the propagation for the harmonic HH65 on axis. The quasiphase-matching effect due to the periodically distributed static electric field, with $\beta=0.005$, is clear in the contribution of the long paths. (b) Evolution of the dipole phase showing the effect of the periodically distributed static electric field on the different path contributions to the harmonics, as indicated. energy $[7,8]$. Furthermore, for relatively intense static electric fields, effects from the breaking of the inversion symmetry in the nonlinear process of the driving laser interacting with the atom or molecule dominate, which results in doubleplateau scenarios and the appearance of even- and odd-order harmonics [9-11].

In the particular case reported in the present study, only very weak electric field strengths have been considered, with which phenomena as those just mentioned above are hardly observed. Instead, the main effect of the weak static electric field considered here is to shift the phase of the electron quantum trajectories in the single-atom high-harmonic-generation process, as it will be shown next.

Figure 4 shows the evolution along the propagation direction of the generated spectral intensity [Fig. 4(a)], as well as the dipole phase [Fig. 4(b)], for the harmonic HH65, which is well in the plateau region (see Fig. 2). This has been computed for the long and short trajectories separately, considering the case without static electric field $(\beta=0)$ and for $\beta=0.005$. In the case of $\beta=0.005$, Fig. 4(a) shows a steplike evolution of the spectral intensity, which is characteristic for yield enhancement by QPM, although this is only observed on the
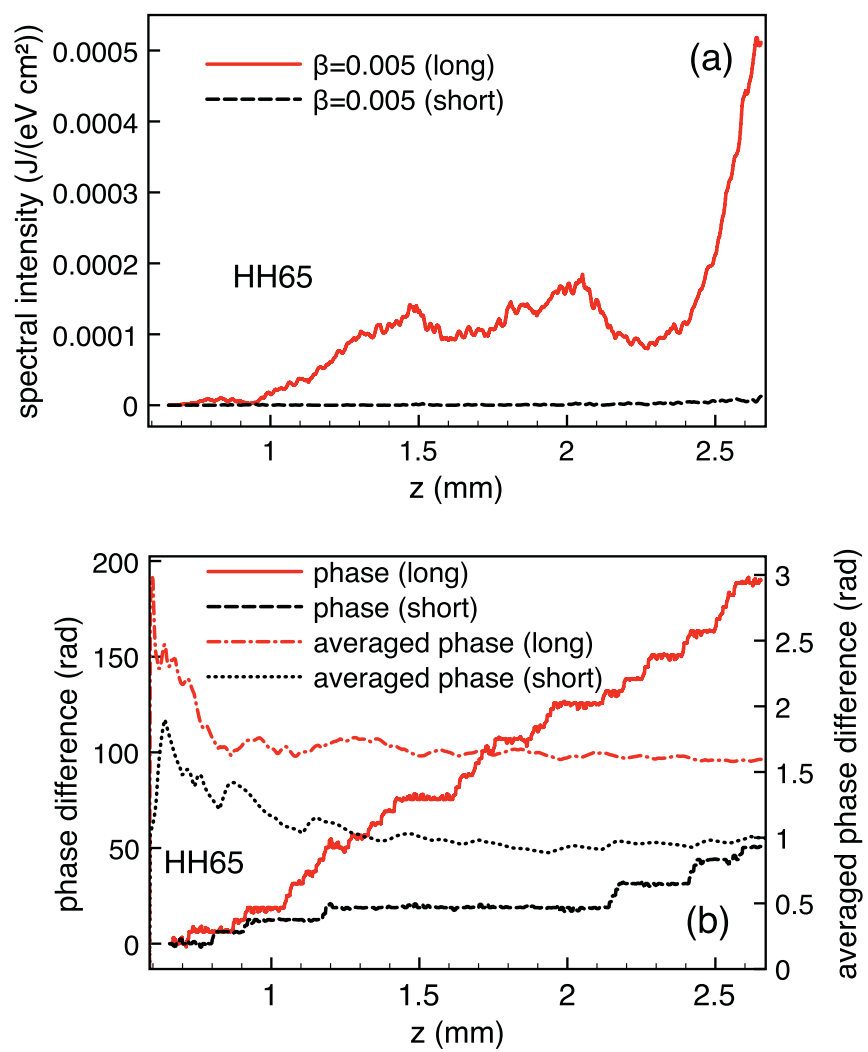

FIG. 5. (Color online) (a) Evolution of the spectral intensity with $\beta=0.005$ for the long- and short-path contributions in the first $2 \mathrm{~mm}$ of the propagation for the harmonic HH65 on axis. (b) Evolution of the difference between the dipole phase for $\beta=$ 0.005 and the one for $\beta=0$, in the first $2 \mathrm{~mm}$ of the propagation, for the long- and short-path contributions (solid and dashed lines). In the right-hand axis the average of the dipole phase difference is calculated at each propagation distance, showing the effect of the faster variation experienced by the quantum phase along the longer electron trajectories (dotted-dashed and dotted lines). 
long-path contribution. This behavior can be understood by studying the phase accumulated by the electron wave packet during its acceleration by the laser field between time of release and time of return, which is the phase of the time-dependent dipole moment as shown in Fig. 4(b). In the regions where the static electric field is present $(\beta=0.005)$, a variation of the dipole phase with respect to the case without static electric field $(\beta=0)$ is present both in the short- and long-path contributions. However, the variations of the phase in the long trajectories is clearly larger. This is expected, since the quantum phase is proportional to the driving field intensity and the duration of the trajectory [12], and thus the longer quantum trajectories experience a faster phase change. Clearly, these more important variations of the dipole phase in the longer trajectories lead to an optimal QPM macroscopic effect, which enhances the yield in the harmonic HH65 on the axis along propagation. Note that some spurious $\pi$ jumps associated with values of the dipole amplitude near zero are also present.

In Fig. 5 the simulations are extended to a propagation distance of $2 \mathrm{~mm}$ in order to obtain a quantitative estimation of the different behavior observed from the contribution to the harmonics generated by the long trajectories and the short ones. Figure 5(a) shows a very different behavior of the propagated yield of the harmonic HH65 on the axis obtained from the longpath contribution compared to the one obtained considering the short paths for $\beta=0.005$. Figure 5(b) shows the evolution of the difference between the dipole phase for $\beta=0.005$ and the one for $\beta=0$ (left-hand axis), and also the average of the dipole phase difference (right-hand axis) — this is, at each propagation distance, all dipole phase differences occurring have been averaged, excluding the null values from the regions where the static electric field is not present. On the one hand, from Fig. 5(b), it is clear that the phase changes are much more important in the case of the long trajectories (solid red/gray line) than in the case of the short ones (dashed black line). On the other hand, the dotted-dashed and dotted lines in Fig. 5(b) show that the averaged magnitude has an asymptotic value of $\approx \pi / 2 \mathrm{rad}$ at a propagation distance of $2 \mathrm{~mm}$ in the case of the long trajectories [dotted-dashed red/gray line in Fig. 5(b)], and a smaller value of $\approx 1 \mathrm{rad}$ in the case of the short ones (dotted black line). From this last result it can be inferred that the phase variations on the long trajectories are mostly able to rephase the dipoles in the regions where $\beta=0.005$, so that in average the interference between the traveling harmonics field and the generated one can be constructive in the regions where the static field is applied, as it is required to obtain a macroscopic QPM effect. As is also clear enough, this is not the case for the short trajectories.

In conclusion, I have presented a coherent quantum control tool for HHG. This tool is based on adding a static-electricfield spatial pattern in the interaction volume of the HHG process. It can be implemented by using the transverse profile

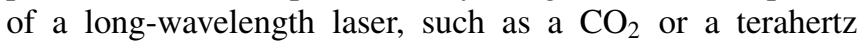
pulsed laser, propagating orthogonal to the driving field. I have studied the case of a periodic uniform weak static electric field distributed along the propagation direction of the driving field. The results show how this particular pattern modifies the phase of the time-dependent dipole moment in the single-atom interaction of the high-harmonics-generation process, leading to a macroscopic QPM effect, which allows to control the yield of the harmonics emitted at different regions of the electromagnetic spectrum. I have shown selectivity on the short and long trajectories in the plateau region, which results in a filtering of the emitted harmonics field. The richness of the effects observed in the single-atom interaction by applying static electric fields, together with the possibility of controlling propagation effects in the generation process by employing different spatial static-electric-field patterns suggests a general scheme for feedback loop control in the attophysics laboratory.

Support from the Spanish Ministry of Education and Science through Project No. FIS2008-06368-C02-02, as well as computer resources from Barcelona Supercomputing Center, is acknowledged.
[1] A. Scrinzi, M. Yu. Ivanov, R. Kienberger, and D. M. Villeneuve, J. Phys. B 39, R1 (2006).

[2] H. Rabitz, R. de Vivie-Riedle, M. Motzkus, and K. Kompa, Science 288, 824 (2000).

[3] C. Serrat and J. Biegert, Phys. Rev. Lett 104, 073901 (2010).

[4] M. Lewenstein, Ph. Balcou, M. Yu. Ivanov, A. L'Huillier, and P. B. Corkum, Phys. Rev. A 49, 2117 (1994).

[5] E. Priori et al., Phys. Rev. A 61, 063801 (2000).

[6] See e.g., S. Coriani, C. Hättig, and A. Rizzo, J. Chem. Phys. 111, 7828 (1999).
[7] M-Q. Bao and A. F. Starace, Phys. Rev. A 53, R3723 (1996).

[8] B. Borca, A. V. Flegel, M. V. Frolov, N. L. Manakov, D. B. Milosevic, and A. F. Starace, Phys. Rev. Lett. 85, 732 (2000).

[9] A. Lohr, W. Becker, and M. Kleber, Laser Physics 7, 615 (1997).

[10] B. Wang, X. Li, and P. Fu, J. Phys. B 31, 1961 (1998).

[11] W. Hong, P. Lu, W. Cao, P. Lan, and X. Wang, J. Phys. B 40, 2321 (2007).

[12] M. B. Gaarde, J. L. Tate, and K. J. Schafer, J. Phys. B 41, 132001 (2008). 